\title{
ARTí́CULO
}

\section{Explotación intensiva de la tintorera Prionace glauca y del marrajo Isurus oxyrinchus: Análisis de la pesquería en el Atlántico noreste entre 2001 y 2016}

Intensive exploitation of blue shark Prionace glauca and shortfin mako Isurus oxyrinchus: Analysis of the fisheries in the North-eastern Atlantic Ocean from 2001 to 2016

\section{Sebastián Biton-Porsmoguer ${ }^{1^{*}}$}

\begin{abstract}
${ }^{1}$ Aix-Marseille University, CNRS/INSU, Toulon University, IRD, Mediterranean Institute of Oceanography (MIO) UM 110, Campus de Luminy, 13288 Marseille, France.*sebastianbiton@gmail.com

Abstract.- Blue shark (Prionace glauca) and shortfin mako (Isurus oxyrinchus) are species exploited by the Spanish and Portuguese longline fleet. They land sharks in the fish market at Vigo (Spain). The number of longliners decreased between 2004 and 2016 (less 15 boats). Landings of shortfin mako have decreased and those of blue shark increased between 2001 and 2016. In accordance with ICCAT informs, the blue shark stock is not overexploited. But it is for shortfin mako in the North-eastern Atlantic Ocean. The sharks landed in a fish market are mainly juvenile, the maturity age being late for both species. The authorities must establish measures to reduce fishing pressure.
\end{abstract}

Key words: Blue shark, shortfin mako, longline fishery, Northeastern Atlantic Ocean

Resumen.- Las tintoreras (Prionace glauca) y los marrajos (Isurus oxyrinchus) son especies objetivo explotadas por los palangreros españoles y portugueses. Las tripulaciones desembarcan los tiburones en la lonja de Vigo (España). La flota palangrera perdió 15 unidades de pesca entre 2004 y 2016. Del 2001 al 2016, las cantidades desembarcadas de marrajos disminuyeron cuando las cantidades de tintoreras subieron notablemente. Según los informes del ICCAT, el stock de tintorera no está sobreexplotado. Sin embargo, el stock del marrajo sí lo estaría en el Atlántico noreste. Los tiburones capturados y vendidos en la lonja son en la gran mayoría, juveniles, siendo la madurez sexual muy tardía para ambas especies. Las autoridades deben tomar medidas para reducir la presión de la pesca ejercida sobre ambas especies.

Palabras clave: Tintorera, marrajo, palangreros, Océano Atlántico noreste

\section{INTRODUCCIÓN}

La zona de estudio que se localiza entre el archipiélago de las Azores y la Península Ibérica se caracteriza por una rica biodiversidad marina (Sobrinho-Gonçalves \& Isidro 2001, Figueiras et al. 2002, Vasco-Ferreira 2011) y es especialmente propicia a la explotación pesquera tanto en alta mar como cerca de la costa. Esta zona es reconocida como un área de migración de la tintorera (Prionace glauca) (Linnaeus, 1758) y del marrajo (Isurus oxyrinchus) Rafinesque, 1809 que provienen del Atlántico oeste (Compagno et al. 2005). Los tiburones en la zona son recurso objetivo y objeto de una intensa explotación por parte de las industrias pesqueras española y portuguesa, en el marco de acuerdos de colaboración entre ambos países. La pesca de la tintorera y del marrajo no está regulada por ninguna medida.

El presente estudio tiene dos principales objetivos: 1) analizar los niveles de desembarques y esfuerzo pesquero de tintorera y marrajo realizados por los palangreros españoles y portugueses en la lonja de Vigo (Galicia, España) durante 16 años, en el periodo comprendido entre 2001 y 2016 y 2) analizar la información biológica colectada durante el 2012 y 2013. 
A partir de los datos obtenidos sobre las flotas palangreras (números de unidades) (Anexo Fig. S1a) y los niveles de desembarques de tiburones (Xunta de Galicia, com. pers) ${ }^{1}$, se calculó las capturas por unidad de esfuerzo (CPUE). Este índice, con los recaudos del caso, puede ser usado como índice de abundancia relativa (Venables \& Dichmont 2004). Los economistas utilizan también la CPUE para analizar la producción global (Kirkley et al. 2004).

En el presente estudio, una unidad de esfuerzo es 'un conjunto de barcos pesqueros utilizando el mismo arte de pesca, que pescan la misma especie y el mismo stock, en la misma zona de explotación y durante el mismo periodo' (Marchal 2008). La flota de estudio utiliza el palangre de superficie con un único modelo de anzuelo, pescan las mismas especies (la tintorera y el marrajo), en la misma zona (entre la Península Ibérica y el archipiélago de las Azores) durante el mismo periodo y no ha conocido mejoras tecnológicas relevantes entre 2001 y 2016 (Xunta de Galicia, com. pers) ${ }^{1}$. Dicho análisis permitirá conocer la variación inter- e intra- anual de los desembarques. El análisis de la información colectada en los muestreos permitirá conocer la fracción de la población explotada, la proporción por sexo, y la relación entre adultos y juveniles en la zona. Los individuos de las especies estudiadas se separan según los sexos o la edad. Las tintoreras juveniles permanecen juntas hasta alcanzar $130 \mathrm{~cm}$ de Longitud Total (LT) y después migran con otros individuos de misma edad y sexo (Compagno et al. 2005). Las hembras pueden también reunirse en la fase de gestación. Los adultos (machos y hembras) solamente se reúnen para acoplarse (Compagno et al. 2005). En el caso del presente estudio, los datos permitirán indicar una observación, en cuanto a la estructura poblacional explotada.

\section{MATERIALES Y MÉtodos}

\section{Desembarques (en TON) y CPUE}

Los datos de desembarques, comprendido en el periodo entre 2001 y 2016, por especie, por año, así como el número de unidades de pesca (entre 2004 y 2016) fueron proporcionados por la Xunta de Galicia (Xunta de Galicia, com. pers $)^{1}$. Los niveles de desembarques por especie (en ton) y el número de unidades de la flota palangrera

${ }^{1}$ Xunta de Galicia, Conselleria do Mar, C/ de San Caetano, S/N, 15781 Santiago de Compostela, España. <https://www.xunta.gal> (número de barcos) que explotan el conjunto de las zonas pesqueras (territoriales y alta mar) en un periodo dado, han permitido calcular la Captura Por Unidades de Esfuerzo (CPUE):

$$
\mathrm{CPUE}=\frac{\text { desembarques por año }(\text { ton })}{\mathrm{n}^{\circ} \text { de unidades de esfuerzo }\left(\mathrm{n}^{\circ} \text { de palangreros }\right)}
$$

\section{ZONAS DE LOS TIBURONES MUESTREADOS}

Fue posible contar con la georreferenciación de los tiburones muestreados en las distintas zonas localizadas entre el archipiélago de las Azores y la Península Ibérica (Fig. 1). La distribución de los tiburones muestreados en las distintas zonas se presenta en la Tabla 1. En 2012, se muestrearon 54 tintoreras y 82 marrajos cerca del archipiélago de las Azores (zonas A, B, C). En 2013, se amplió la zona de muestreo con 122 tiburones, 55 tintoreras y 67 marrajos, capturados entre el archipiélago de las Azores y la Península Ibérica (zonas D, G, F) y al norte de Galicia (zona E) (Fig. 1).

\section{MEDIDAS BIOMÉTRICAS Y SEXO}

Se obtuvieron los datos biométricos de los individuos muestreados en la lonja de Vigo. Se consignó la longitud total (LT) y el peso total (kg), cuando fue posible. Se ajustaron correlaciones no lineales entre la longitud total (cm) y el peso (kg) para ambas especies.

Tabla 1. Distribución del número de individuos muestreados por especie y por zona de muestreo en 2012 y 2013. Ausencia de muestras vs individuos capturados en la zona (-). Zona no explorada (0) / Distribution of sampled sharks per species and per zone between 2012 and 2013. Not samples vs individuals catch in the zone (-). Zone not explored (0)

\begin{tabular}{lcccccccc}
\hline Zonas & A & B & C & D & E & F & G & Total \\
\hline 2012 & & & & & & & & \\
Tintorera & 0 & 26 & 28 & 0 & 0 & 0 & 0 & 54 \\
Marrajo & 35 & 6 & 41 & 0 & 0 & 0 & 0 & 82 \\
$\quad$ Total 2012 & 35 & 32 & 69 & 0 & 0 & 0 & 0 & 136 \\
2013 & & & & & & & & \\
Tintorera & 0 & 18 & 0 & - & 34 & - & 3 & 55 \\
Marrajo & 0 & 16 & 0 & 37 & - & 11 & 3 & 67 \\
$\quad$ Total 2013 & 0 & 34 & 0 & 37 & 34 & 11 & 6 & 122 \\
Total & 35 & 66 & 69 & 37 & 34 & 11 & 6 & 258 \\
\hline
\end{tabular}

Zona A: $37^{\circ} \mathrm{N}-32^{\circ} \mathrm{O}$; Zona B: $42^{\circ} \mathrm{N}-22^{\circ} \mathrm{O}$; Zona C: $38^{\circ} \mathrm{N}-20^{\circ} \mathrm{O}$; Zona D: $33^{\circ} \mathrm{N}-30^{\circ} \mathrm{O}$; Zona E: $45^{\circ} \mathrm{N}-9^{\circ} \mathrm{O}$; Zona F: $40^{\circ} \mathrm{N}-11^{\circ} \mathrm{O}$; Zona G: $36^{\circ} \mathrm{N}-12^{\circ} \mathrm{O}$ 


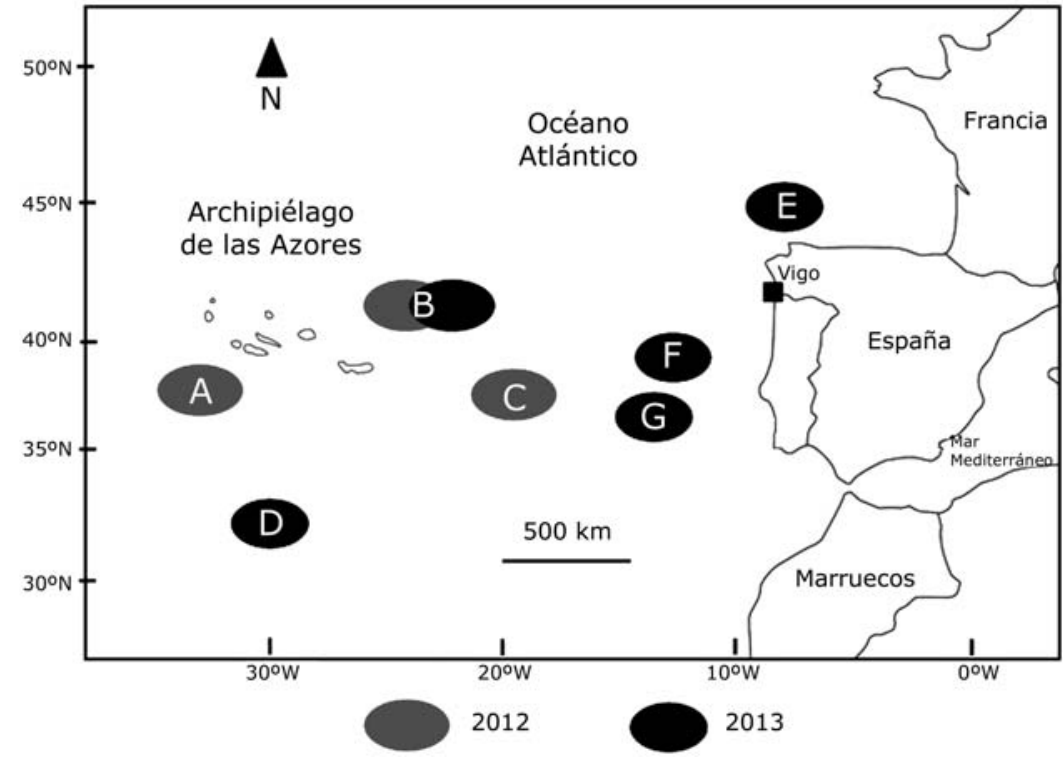

Figura 1. Zonas de muestreo entre el archipiélago de las Azores y la Península Ibérica / Sampling areas between Azores Archipelago and Iberian Peninsula
El sexo de cada individuo se ha determinado observando la presencia o ausencia de los pterigopodios (órganos sexuales masculinos externos). En este estudio, la proporción sexual está representada por el número de hembras sobre el número de machos. La edad de madurez sexual de las especies muestreadas varía según las distintas poblaciones (Compagno et al. 2005, Valeiras \&
Abad 2010a). En el Atlántico norte, las tintoreras hembras alcanzan la madurez sexual a los $200 \mathrm{~cm}$ LT y los machos a $180 \mathrm{~cm}$ LT (Moreno 2004). En cuanto a los marrajos, las hembras alcanzan la madurez sexual a los $280 \mathrm{~cm}$ LT y los machos a los $195 \mathrm{~cm}$ LT (Moreno 2004). A partir de los datos de madurez sexual, los individuos fueron clasificados en distintas categorías de tallas (Tabla 2).

Tabla 2. Número de tintoreras y marrajos muestreados por clases de talla (cm), años (2012 y 2013) y estación (invierno/primaveraverano/otoño) / Number of blue sharks and shortfin makos sampled per size classes (cm), years (2012 and 2013 ) and seasons (winter/spring-summer/autumn)

\begin{tabular}{crrrrr}
\hline $\begin{array}{c}\text { Clases de } \\
\text { talla } \\
(\mathrm{cm})\end{array}$ & $\begin{array}{c}\text { Invierno/ } \\
\text { Primavera } \\
2012\end{array}$ & $\begin{array}{c}\text { Verano/ } \\
\text { Otoño } \\
2012\end{array}$ & $\begin{array}{c}\text { Invierno/ } \\
\text { Primavera } \\
2013\end{array}$ & $\begin{array}{c}\text { Verano/ } \\
\text { Otoño } \\
2013\end{array}$ & Total \\
\hline $\begin{array}{c}\text { Tintorera } \\
70-99\end{array}$ & 1 & 0 & & & \\
$100-129$ & 15 & 0 & 5 & 1 & 26 \\
$130-159$ & 6 & 3 & 5 & 3 & 17 \\
$160-199$ & 3 & 9 & 4 & 3 & 19 \\
$\geq 200$ & 1 & 16 & 0 & 4 & 21 \\
Total & 26 & 28 & 18 & 37 & 109 \\
Marrajo & & & & & \\
$\leq 99$ & 0 & 1 & 0 & 0 & 1 \\
$100-119$ & 20 & 14 & 4 & 6 & 44 \\
$120-139$ & 0 & 9 & 27 & 2 & 38 \\
$140-159$ & 6 & 4 & 0 & 7 & 17 \\
$160-179$ & 10 & 5 & 4 & 4 & 23 \\
$180-199$ & 2 & 4 & 2 & 6 & 14 \\
$\geq 200$ & 3 & 4 & 3 & 2 & 12 \\
Total & 41 & 41 & 40 & 27 & 149 \\
\hline
\end{tabular}




\section{Tratamiento ESTAdístico DE LOS DATOS}

Con el objetivo de comparar la talla de los individuos muestreados por sexo, entre las estaciones (inviernoprimavera y verano-otoño), entre años y entre las distintas zonas de pesca y entre macho y hembra se realizaron pruebas estadísticas con el programa STATISTICA versión 9.1. El ajuste de modelos potenciales obtenido entre ambos factores permitió elegir la talla o el peso. Sin embargo, ya que algunos individuos se pesaron eviscerados y otros enteros, es el factor talla que fue elegido para el estudio comparativo de los datos biométricos. La normalidad y homogeneidad de las variables se comprobaron realizando los tests de Shapiro y de Levene, respectivamente. Cuando la variable

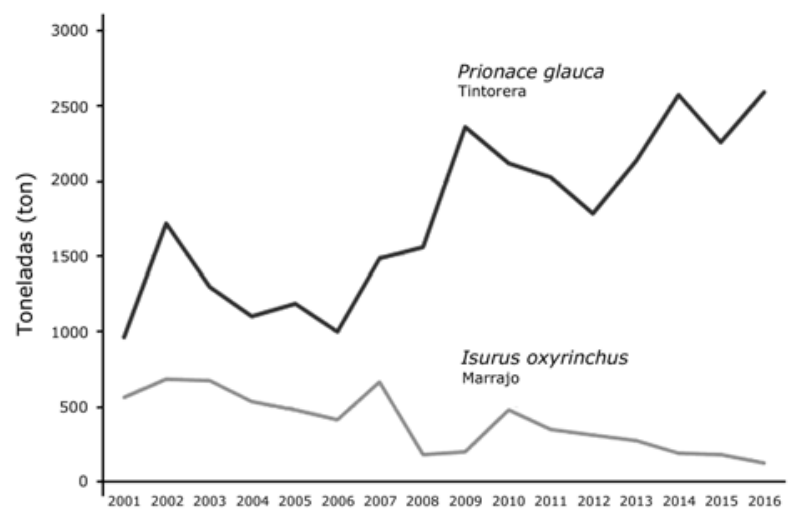

Figura 2. Evolución de los desembarques totales en toneladas (ton) de tintorera y marrajo en Vigo, España entre 2001 y 2016. Fuente: Xunta de Galicia / Evolution of landing quantities in Tonnes (ton) of blue shark and shortfin mako in Vigo, Spain between 2001 to 2016. Source: Xunta de Galicia



Figura 3. Evolución del número de palangreros de superficie que desembarcan tintoreras y marrajos en Vigo, España, entre 2004 y 2016. Fuente: Xunta de Galicia / Evolution of longliner number landing blue sharks and shortfin makos in Vigo, Spain between 2004 to 2016. Source: Xunta de Galicia estudiada no se ajustó a la distribución normal, se realizó el test no paramétrico de Mann-Whitney (U), para ambas especies, con la comparación de los datos entre 2012 y 2013 de las estaciones y entre machos y hembras. En cuanto a las zonas de muestreos, la variable considerada tampoco se ajustó a una distribución normal. Como consecuencia, la comparación se realizó mediante el test no paramétrico de Kruskal-Wallis. Todos los tests estadísticos en este estudio se realizaron conforme a la metodología indicada por Scherrer (2007).

\section{Resultados}

ANÁlisis de los DATOS DE DESEMBARQues, NÚMERo DE EMBARCACIONES Y CPUE

Se observaron importantes fluctuaciones en las cantidades desembarcadas de tintoreras y marrajos (en ton) (Fig. 2). Los desembarques de las tintoreras aumentaron en el periodo analizado. Dichos desembarques pasan de 964 ton en 2001 a más de 2.591 ton en 2016, lo que significa un aumento de más del 165\%. Los desembarques de marrajo, a pesar de dos aumentos en el 2007 y 2010, disminuyeron pasando de 560 ton en 2001 a 119 ton en 2016, lo que supone una disminución de cerca del $80 \%$ en 16 años.

Los palangreros explotan aguas nacionales e internacionales. El número de palangreros de superficie que explotan aguas internacionales (aguas que se sitúan a más de $370 \mathrm{~km}$ de la costa), aumentó de 69 a 87 unidades, entre 2004 y 2009. En 2016, el número de unidades registradas disminuye a 68 , según datos comunicados por la Xunta de Galicia (com. pers) ${ }^{1}$ (Fig. 3). Las CPUE calculadas como ton por embarcación, de las tintoreras para las flotas palangreras que explotan aguas nacionales e internacionales, muestran una tendencia creciente entre 2004 y 2016 (Fig. 5), la CPUE aumentó de 8 ton/embarcación en 2004 a más de 21 ton/embarcación en 2016. Respecto al marrajo, las CPUE disminuyeron, situándose de 4 ton anuales a apenas 1 ton anual por palangrero durante el período estudiado (Fig. 4).

\section{ANÁLISIS DE LOS MUESTREOS REALIZADOS}

Los ajustes entre los modelos potenciales entre el peso (Peso total, kg) y la talla (Longitud total, cm) de los individuos fueron elevados: $\mathrm{R}^{2}=0,9575$ para las tintoreras (individuos enteros) (Fig. 5a), $\mathrm{R}^{2}=0,9504$ para los marrajos enteros (Fig. 5b) y $\mathrm{R}^{2}=0,9129$ para los marrajos eviscerados (Fig. 5c). 


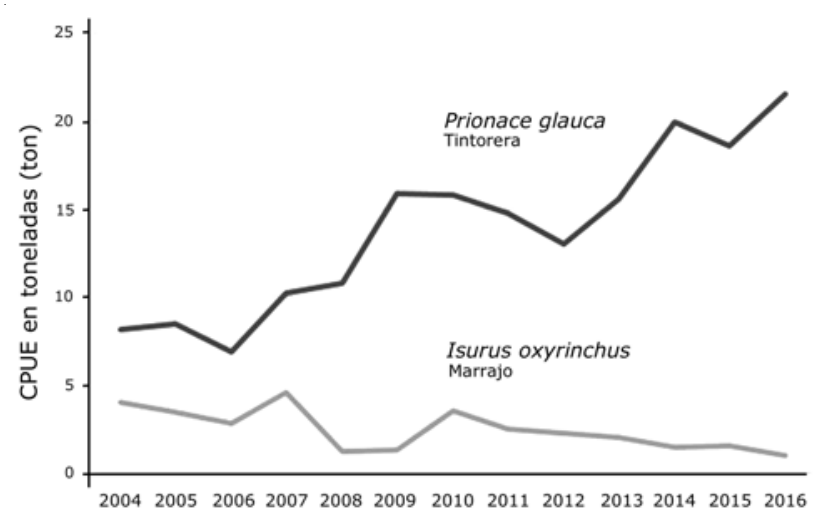

Figura 4. Evolución de la CPUE de la tintorera y del marrajo, en toneladas (ton) por palangrero, desde el 2004 al 2016 / Evolution of CPUE for blue shark and shortfin mako, in Tonnes (ton), per longliner boat, from 2004 to 2016

a)

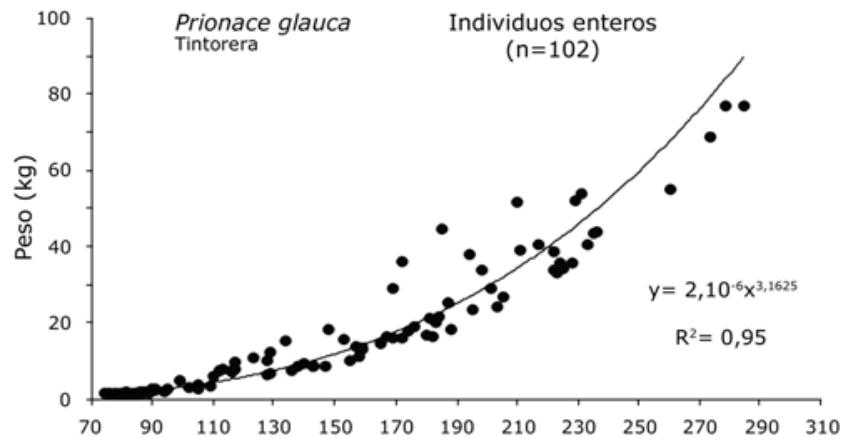

b)

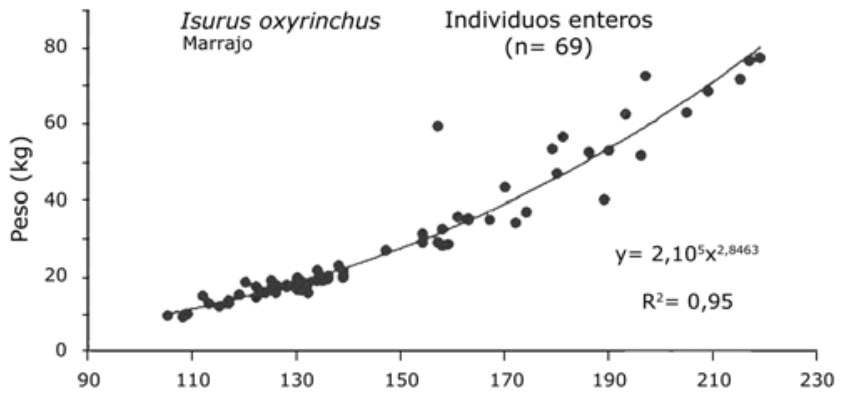

c)

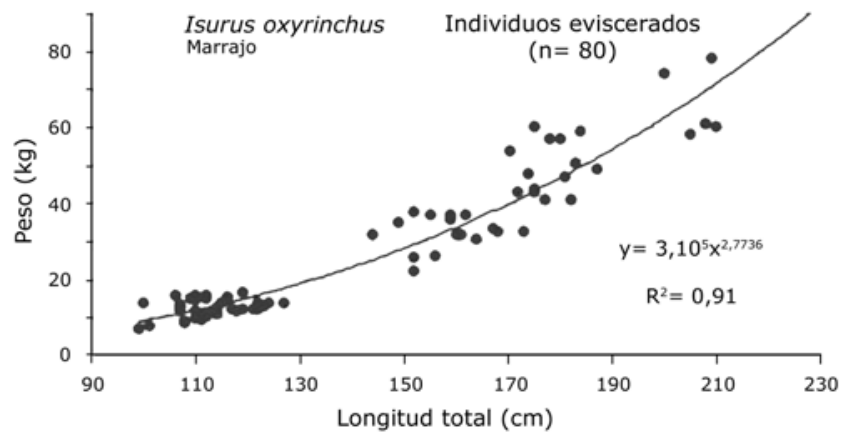

Figura 5. Representación gráfica de los modelos potenciales entre la talla (Longitud total, en $\mathrm{cm}$ ) y el peso (Peso, $\mathrm{kg}$ ). a) Tintoreras enteras, b) Marrajos enteros, c) Marrajos eviscerados / Graphs of power relation between length (Total length, in $\mathrm{cm}$ ) and weight (weight, in kg). a) Blue shark not eviscerated, b) Shortfin makos not eviscerated, c) Shortfin makos eviscerated 
Tabla 3. Número de individuos muestreados de adultos y juveniles de tintorera y marrajo, por años (2012 y 2013) y estación (invierno/ primavera-verano/otoño) / Distribution between adults and juveniles of blue sharks and shortfin makos sampled, per years (2012 and 2013) and seasons (winter/spring-summer/autumn)

\begin{tabular}{|c|c|c|c|c|c|c|}
\hline \multirow{2}{*}{ Especie/Estación } & \multicolumn{2}{|c|}{2012} & \multicolumn{2}{|c|}{2013} & \multicolumn{2}{|c|}{ Total } \\
\hline & Adultos & Juveniles & Adultos & Juveniles & Adultos & Juveniles \\
\hline \multicolumn{7}{|l|}{ Tintorera } \\
\hline Invierno/Primavera & 1 & 25 & 1 & 17 & 2 & 42 \\
\hline Verano/Otoño & 22 & 6 & 6 & 31 & 28 & 37 \\
\hline Total & 23 & 31 & 7 & 48 & 30 & 79 \\
\hline \multicolumn{7}{|l|}{ Marrajo } \\
\hline Invierno/Primavera & 1 & 40 & 1 & 39 & 2 & 79 \\
\hline Verano/Otoño & 3 & 38 & 4 & 23 & 7 & 61 \\
\hline Total & 4 & 78 & 5 & 62 & 9 & 140 \\
\hline
\end{tabular}

Tabla 4. Número de individuos muestreados machos y hembras de tintorera y marrajo, por estación (invierno/primavera-verano/ otoño), años (2012 y 2013) y proporción sexual (hembra/macho)/ Distribution between male and female of blue sharks and shortfin makos sampled, per seasons (winter/spring-summer/ autumn) and sex ratio (female/male)

\begin{tabular}{|c|c|c|c|c|c|c|c|}
\hline \multirow{2}{*}{ Especie/Estación } & \multicolumn{2}{|c|}{2012} & \multicolumn{2}{|c|}{2013} & \multicolumn{2}{|c|}{ Total } & \multirow{2}{*}{$\begin{array}{l}\text { Proporción } \\
\text { sexual }\end{array}$} \\
\hline & M & $\mathrm{H}$ & M & $\mathrm{H}$ & M & $\mathrm{H}$ & \\
\hline \multicolumn{8}{|l|}{ Tintorera } \\
\hline Invierno/Primavera & 5 & 21 & 5 & 13 & 10 & 34 & 3,4 \\
\hline Verano/Otoño & 23 & 5 & 17 & 20 & 40 & 25 & 0,6 \\
\hline Total & 28 & 26 & 22 & 33 & 50 & 59 & 1,2 \\
\hline \multicolumn{8}{|l|}{ Marrajo } \\
\hline Invierno/Primavera & 10 & 31 & 22 & 18 & 32 & 49 & 1,5 \\
\hline Verano/Otoño & 17 & 24 & 15 & 12 & 32 & 36 & 1,1 \\
\hline Total & 27 & 55 & 37 & 30 & 64 & 85 & 1,3 \\
\hline
\end{tabular}

$\mathrm{M}=$ macho, $\mathrm{H}=$ hembra

\section{DiSTRIBUCIÓN ENTRE ADULTO Y JUVENIL Y ENTRE MACHO Y HEMBRA}

La pesquería captura individuos juveniles, tanto para la tintorera como para el marrajo. Entre las 109 tintoreras, 30 fueron adultas y 79 juveniles. En el marrajo, la relación fue más notoria: 9 adultos de los 149 marrajos muestreados (Tabla 3).

Para la tintorera, la relación hembra / macho fue equilibrada: 59 hembras y 50 machos. Para el marrajo, las hembras dominan claramente los individuos muestreados: 85 hembras y 64 machos (Tabla 4).

\section{INFLUENCIA DE LOS FACTORES ANALIZADOS SOBRE LA TALLA (LT) DE LA TINTORERA}

Hubo una diferencia significativa de talla entre los machos y las hembras $(P=0,024)$, la longitud total de los machos fue más elevada en media, que las hembras $(+29,8 \mathrm{~cm})$ (Tabla 5).

Los tests realizados no mostraron diferencias significativas con ese factor entre los individuos muestreados en invierno-primavera y verano-otoño $(P=$ $0,341)$. Sin embargo, se observó una diferencia significativa de talla de los individuos muestreados entre 2012 y $2013(P<0,001)$, siendo los individuos más grandes de 60 cm de media en 2012 comparado con 2013 (Tabla 5).

Tabla 5. Tallas medias y desviación estándar de la longitud total (LT, cm) de las tintoreras muestreadas por sexo, estación (invierno/primavera-verano/otoño) y años (2012/2013). Test Mann-Whitney (valor $\boldsymbol{P}$ ) / Median length and standard deviation of the total length (LT, cm) of the blue shark sampled per sex, seasons (winter/springsummer/autumn) and years (2012 and 2013). Test Mann-Whitney ( $P$-value)

\begin{tabular}{lcccccc}
\hline & Macho & Hembra & $\begin{array}{c}\text { Invierno/ } \\
\text { Primavera }\end{array}$ & $\begin{array}{c}\text { Verano/ } \\
\text { Otoño }\end{array}$ & 2012 & 2013 \\
\hline Talla (LT) & 161,9 & 132,1 & 131,1 & 154,0 & 178,9 & 118,6 \\
Valor $P$ & $(8,9)$ & $(8,9)$ & $(6,3)$ & $(8,1)$ & $(7,4)$ & $\begin{array}{c}(6,1) \\
(0,341\end{array}$ \\
\hline
\end{tabular}

ns $=$ no significativo 
Tabla 6. Tallas medias y desviación estándar de la longitud total (LT, $\mathrm{cm})$ de las tintoreras por zonas de muestreo. Test Kruskal-Wallis (valor $\boldsymbol{P}$ ) / Median length and standard deviation of the total length $(\mathrm{LT}, \mathrm{cm})$ for blue shark per zone. Test Kruskal-Wallis ( $P$-value)

\begin{tabular}{ccccc}
\hline Zonas & $\mathrm{B}(\mathrm{n}=44)$ & $\mathrm{C}(\mathrm{n}=28)$ & $\mathrm{E}(\mathrm{n}=34)$ & $\mathrm{G}(\mathrm{n}=3)$ \\
\hline B & & $* * *$ & $* *$ & $\mathrm{~ns}$ \\
$\mathrm{C}$ & $\cdot$ & & $* * *$ & $\mathrm{~ns}$ \\
E & $\cdot$ & $\cdot$ & & $*$ \\
G & $\cdot$ & $\cdot$ & $\cdot$ & \\
Talla (LT) & $133,1(29,0)$ & $208,8(38,9)$ & $105,6(44,9)$ & $190,3(27,6)$ \\
\hline
\end{tabular}

* $P<0,05 ; * * P<0,01 ; * * * P<0,001$

$\mathrm{ns}=$ no significativo, $\mathrm{n}=$ número de individuos

La talla de las tintoreras mostró diferencias significativas $(P<0,001)$ entre distintas zonas de muestreo (Tabla 6). Dichas diferencias aparecen entre las zonas B y C (la primera situándose cerca del archipiélago de las Azores y la segunda entre el archipiélago y la Península Ibérica); entre las zonas B y E (cerca del archipiélago de las Azores y al norte de Galicia, cerca del litoral); entre las zonas C y E (entre el archipiélago y la Península Ibérica y al norte de Galicia) y entre las zonas E y G (entre el norte de Galicia y la zona muestreada más cerca del sur de Portugal). Ninguna diferencia se observó entre las zonas B y $\mathrm{G}$ y entre las zonas C y G (Tabla 6).

\section{INFLUENCIA DE LOS FACTORES ANALIZADOS SOBRE LA TALLA (LT) DEL MARRAJO}

También hubo una diferencia significativa de talla, estadísticamente hablando, entre los machos y las hembras en el marrajo $(P=0,007)$. La talla media no presentó una diferencia significativa entre las estaciones $(P=0,286)$ pero sí presentó diferencias entre años; los marrajos muestreados en 2013 fueron superiores de $6 \mathrm{~cm}$ (de media) en comparación con los marrajos muestreados en $2012(P=0,036)$ (Tabla 7). La única diferencia significativa obtenida entre las zonas muestreadas se observó entre las zonas A y B, ambas situadas cerca del archipiélago de las Azores, donde se capturaron los marrajos más pequeños (zona A) y más grandes (zona B) $(P<0,008)$ (Tabla 8).

Tabla 7. Tallas medias y deviación estándar de la longitud total $(\mathrm{LT}, \mathrm{cm})$ de los marrajos muestreados por sexo, estación y años (2012/2013). Test Mann-Whitney (valor $P$ ) / Median length and standard deviation of the total length (LT, Cm) for shortfin mako per sex, seasons and years (2012/2013). Test Mann-Whitney (P-value)

\begin{tabular}{lcccccc}
\hline & Macho & Hembra & $\begin{array}{c}\text { Invierno/ } \\
\text { Primavera }\end{array}$ & $\begin{array}{c}\text { Verano/ } \\
\text { Otoño }\end{array}$ & 2012 & 2013 \\
\hline Talla (LT) & 151,8 & 138,6 & 139,9 & 148,8 & 141,4 & 147,1 \\
Valor $P$ & $(4,4)$ & $(3,4)$ & $\begin{array}{c}(3,5) \\
(4,2)\end{array}$ & $\begin{array}{c}(4,3) \\
(3,8)\end{array}$ & $(3,9)$ \\
\hline
\end{tabular}

ns= no significativo

Tabla 8. Tallas medias y desviación estándar de la longitud total (LT, cm) de los marrajos por zona de muestreo. Test Kruskal-Wallis (valor $P$ ) / Median length and standard deviation of the total length $(\mathrm{LT}, \mathrm{cm})$ for shortfin mako per zone. Test Kruskal-Wallis ( $P$-value)

\begin{tabular}{ccccccc}
\hline Zonas & $\begin{array}{c}\text { A } \\
(\mathrm{n}=35)\end{array}$ & $\begin{array}{c}\mathrm{B} \\
(\mathrm{n}=22)\end{array}$ & $\begin{array}{c}\mathrm{C} \\
(\mathrm{n}=41)\end{array}$ & $\begin{array}{c}\mathrm{D} \\
(\mathrm{n}=37)\end{array}$ & $\begin{array}{c}\mathrm{F} \\
(\mathrm{n}=11)\end{array}$ & $\begin{array}{c}\mathrm{G} \\
(\mathrm{n}=3)\end{array}$ \\
\hline $\mathrm{A}$ & & $* *$ & $\mathrm{~ns}$ & $\mathrm{~ns}$ & $\mathrm{~ns}$ & $\mathrm{~ns}$ \\
$\mathrm{~B}$ & $\cdot$ & & $\mathrm{ns}$ & $\mathrm{ns}$ & $\mathrm{ns}$ & $\mathrm{ns}$ \\
$\mathrm{C}$ & $\cdot$ & $\cdot$ & & $\mathrm{ns}$ & $\mathrm{ns}$ & $\mathrm{ns}$ \\
$\mathrm{D}$ & $\cdot$ & $\cdot$ &. & & $\mathrm{ns}$ & $\mathrm{ns}$ \\
$\mathrm{F}$ & $\cdot$ & $\cdot$ & $\cdot$ & $\cdot$ & & $\mathrm{ns}$ \\
G & $\cdot$ &. &. &. &. & \\
Talla (LT) & 136,1 & 162,4 & 142,5 & 137 & 157,5 & 156 \\
& $(33,3)$ & $(22,1)$ & $(32,6)$ & $(30,5)$ & $(36,1)$ & $(52,0)$ \\
\hline
\end{tabular}

$* *=P<0,05 . \mathrm{ns}=$ no significativo, $\mathrm{n}=$ número de individuos 


\section{Discusión}

\section{DESEMBARQues DE TINTORERAS Y MARRAJOS POR LOS} PALANGREROS

Los resultados señalan una tendencia al alza en cuanto a la explotación de la tintorera, con un aumento de la CPUE. Por el contrario, hay una disminución de la CPUE para el marrajo durante el periodo del estudio. La pesca no es el único factor que puede explicar las variaciones observadas respecto a las capturas de los tiburones. Dichas fluctuaciones podrían explicarse también por factores endógenos o exógenos al sistema marino tales como el reclutamiento, la migración, las interacciones con las otras especies o la variabilidad climática que ocurre en las zonas de captura. Sin embargo, no se dispone de datos sobre estos parámetros. No obstante, las variaciones explicadas por los desplazamientos migratorios han sido observadas para otras especies tales como el atún rojo (Fromentin 2006).

En la zona de estudio, la evaluación del stock de los tiburones es realizada por el ICCAT (la Comisión Internacional para la Conservación del Atún Atlántico). En el 2008 y 2015, el ICCAT estimó a partir de modelos de producción excedentaria, modelos estructurados por edad y modelos sin capturas, que las cantidades de extracción actuales de tintorera están por debajo del Rendimiento Máximo Sostenible (RMS). Estos análisis indican que los stocks de tintorera no estarían sobrexplotados (Anón 2009, Valeiras \& Abad 2010a, ICCAT 2014-2015). Por el contrario, el ICCAT considera, que los stocks de marrajo en el Atlántico norte, estuvieron posiblemente sobrexplotados según la evolución histórica de las CPUE (Valeiras \& Abad 2010b) y los niveles de extracción actuales de marrajos estarían por encima del RMS. Según algunos autores, la tendencia de las CPUE estaría indicando un agotamiento del stock de más del 50\% o más (Valeiras \& Abad 2010b). El presente trabajo presenta también una disminución de la CPUE durante el periodo y en la zona de estudio (Fig. 4). Sin embargo, los últimos informes indican grandes incertidumbres en cuanto al estado del stock debido principalmente a una falta de datos biológicos de la especie en la zona de explotación (ICCAT 2014-2015, 2017). El agotamiento del stock confirmado por el ICCAT en 2010 explicaría 1) la fuerte disminución de las capturas de marrajo observada durante el periodo el estudio y 2) la reducción de unidades de pesca observada estos últimos años.
Las características reproductivas de las 2 especies de tiburones estudiadas, con edades de madurez sexual tardías en ambos casos, hacen que sean muy vulnerables en caso de sobreexplotación. Las autoridades competentes (Ministerio de Agricultura, Pesca, Alimentación y Medioambiente, Consellería do Mar de la Xunta de Galicia) deberían tomas las medidas para reducir la presión ejercida por los palangreros sobre las especies explotadas (reducir los niveles de captura y desembarques). Dichas medidas podrían ser, por ejemplo, reducir la flota pesquera, limitar el número de anzuelos permitidos por barco o imponer una cuota y/o talla límite. El objetivo es mantener el nivel de explotación sostenible a largo plazo manteniendo niveles de captura soportable para la biomasa de ambas especies y de rentabilidad aceptable para los pescadores.

\section{DATOS BIOMÉTRICOS DE LA TINTORERA Y DEL MARRAJO}

El resultado obtenido de los muestreos señaló que los juveniles superaban los adultos tanto para la tintorera como para el marrajo. La regulación de la explotación es aún más importante y necesaria ya que la gran mayoría de los individuos aún no se han reproducido. Algunos trabajos sobre los marrajos confirman también una escasa presencia de individuos adultos, tanto en el Atlántico noroeste (Casey \& Kohler 1992) como en el Atlántico noreste (Mejuto 1985). Los datos disponibles sobre la población de las tintoreras en Atlántico noreste confirman también la dominancia de los juveniles, tanto para los machos como para las hembras (Valeiras \& Abad 2010a). Los individuos juveniles dominan claramente los individuos capturados con una amplia amplitud de tallas (74,5 > $284 \mathrm{~cm}$ para la tintorera, $99>216 \mathrm{~cm}$ para el marrajo).

La presencia de individuos de muy pequeña talla a la venta señala que algunas zonas explotadas corresponden a zonas de parición (Anexo Fig. S1b). En efecto, las observaciones de los individuos en venta en la lonja de Vigo en los periodos de muestreo y de los individuos capturados durante los embarques realizados permiten decir que la gran mayoría de los tiburones capturados no han alcanzado la madurez sexual y son juveniles.

Aunque el número de individuos es bajo, 109 tintoreras y 149 marrajos, representan dos años de muestreo, 2012 y 2013 y confirman la observación indicada en la literatura (Clarke et al. 1996, Maia et al. 2006). La captura de individuos juveniles también se explica por las características del arte de pesca utilizado y por el modelo 
de palangreros. El palangre de superficie utilizado solo permite la captura de individuos de pequeña y mediana talla. Los individuos adultos, de más de 2 metros, pueden romper la línea por la presión y la fuerza, y los dientes del tiburón pueden cortar la línea. Para evitar ese inconveniente, algunas veces los pescadores instalan un cable de acero en la línea hasta el anzuelo. Además, la mayoría de los palangreros no están equipados para izar a bordo tiburones de más de 2 metros (una grúa presente en algunos barcos lo permite). Los individuos juveniles dominan claramente los individuos capturados con una amplia amplitud de tallas (74,5 > $284 \mathrm{~cm}$ para la tintorera, $99>216 \mathrm{~cm}$ para el marrajo). Hay que resaltar también la dominancia de las hembras en comparación con los machos en el muestreo realizado, sobre todo para el marrajo. Las poblaciones de las especies estudiadas se separan según la talla y el sexo varias veces a lo largo de sus vidas (Valeiras \& Abad 2010a). En principio, para ambas especies, los individuos juveniles, machos y hembras, viven juntos hasta alcanzar la edad adulta (Compagno et al. 2005). Sin embargo, según Mejuto \& Garcés (1984), entre la Península Ibérica y el archipiélago de las Azores, la población de marrajos estaría dominada por un 71\% de machos de más de $200 \mathrm{~cm}$ FL (largo de horquilla). Los estudios recientes realizados en el Atlántico norte sobre el régimen alimentario de la tintorera (McCord \& Campana 2003, Mendonça 2009) y del marrajo (Maia et al. 2006) solo indican la proporción sexual para los individuos cuyo estómago contiene presas. La dominancia de las hembras en comparación con los machos se explica por el muestreo realizado. Los tests estadísticos han mostrado que la talla media de los individuos era significativamente diferente según el sexo. Los individuos machos presentaban tallas medias superiores que las hembras.

Dichos resultados se explican por los individuos machos más numerosos que las hembras. En efecto, entre las 30 tintoreras adultas, 24 eran machos con una longitud total superior a $180 \mathrm{~cm}$. La tendencia es la misma para el marrajo, 8 de los 9 adultos medían más de 195 cm. No hubo diferencias significativas entre estaciones en las tallas de las tintoreras y los marrajos muestreados (entre invierno-primavera y verano-otoño). La influencia de la variación estacional de las temperaturas de las aguas superficiales no parece afectar las poblaciones de los tiburones capturados. Pueden permanecer todo el año en la zona de explotación. La tintorera vive en aguas con temperaturas comprendidas entre 7 y $25^{\circ} \mathrm{C}$ (Compagno et al. 2005). El marrajo es una especie endotérmica, capaz de mantener a una misma temperatura su cuerpo cual sea la temperatura del agua. Ambas especies consumen un amplio espectro de presas (cefalópodos y teleósteos) que les permite también permanecer todo el año en la zona de explotación (Biton-Porsmoguer 2015).

La diferencia significativa de talla media, de más de 60 $\mathrm{cm}$, aparece entre las tintoreras capturadas entre 2012 y 2013. Dicha diferencia se explica por una presencia más importante de individuos adultos muestreados en 2012 (22 adultos en 2012 y solamente 7 en 2013). Los resultados indican que los marrajos muestreados en 2013 presentan una talla media superior a los marrajos muestreados en 2012. La diferencia es significativa pero menos que para las tintoreras (solo $6 \mathrm{~cm}$ ). Las diferencias indicadas, entre 2012 y 2013, pueden explicarse por el muestreo y no corresponden a criterios biológicos tales como el crecimiento de las especies estudiadas (aumento de la talla de un año a otro de individuos de una misma cohorte). En ese estudio, no se puede considerar el efecto del crecimiento de los individuos sobre esa diferencia de talla inter-anual ya que no se conoce la evolución temporal de las clases de tallas y edades de las poblaciones y de sus migraciones.

Diferencias significativas con las tallas medias de las tintoreras también se observaron entre las distintas zonas de muestreo. Dichos resultados muestran una gran disparidad de talla entre las zonas. La diferencia supera los $100 \mathrm{~cm}$ entre la talla del individuo más pequeño y el más grande. Los individuos sub-adultos y adultos se capturan en alta mar (zonas C y G) y los individuos juveniles se capturan cerca del litoral (zonas B y E). Según Vandeperre et al. (2014), en la tintorera, los recién nacidos permanecen hasta dos años cerca del litoral. Cuando alcanzan $130 \mathrm{~cm}$ aproximadamente, y con una mayor capacidad para nadar, las tintoreras se alejan del litoral, y pueden alimentarse de presas más móviles (Stevens 1976, Muñoz-Chápuli 1984). En cuanto al marrajo, la distribución de los individuos en las zonas muestreadas es más homogénea. Las tallas medias obtenidas por zonas indican diferencias menores que la obtenida con las tintoreras. Los marrajos, tanto pequeños como grandes se capturan cerca del archipiélago de las Azores. La abundancia de presas potenciales asociada a una migración importante desde el nacimiento, son posibles explicaciones.

En conclusión, la explotación de los tiburones pelágicos implica para el sector pesquero español y portugués una fuente de ingresos importante. Cada año, se comercializan en Vigo cerca de 1760 ton de tintorera y 
más de 392 ton de marrajo. En 2016, 120 palangreros se dedicaban a la explotación de estas especies con un notable papel socioeconómico en la región gallega. Los muestreos realizados durante los meses de inviernoprimavera y durante los meses de verano-otoño permitieron estudiar individuos tanto capturados cerca de la costa como en alta mar, cerca del archipiélago de las Azores y cerca de la Península Ibérica. Las tintoreras y marrajos desembarcados en Vigo son, en gran mayoría, individuos juveniles que aún no se han reproducido. El carácter selectivo del palangre de superficie, asociado al muestreo y las características de la zona de estudio, explican probablemente las diferencias obtenidas entre los distintos factores analizados. Las tintoreras con tallas superiores se sitúan más en alta mar cuando los individuos más pequeños se capturaron más cerca de la costa. Sin embargo, no se puede decir lo mismo del marrajo, que presenta una distribución más homogénea entre los individuos más grandes y más pequeños. Tampoco se ha observado una diferencia de distribución entre las dos estaciones estudiadas confirmando la presencia de estas especies durante todo el año. Los análisis de las CPUE muestran estos últimos años (2004-2016) un aumento significativo de los desembarques para la tintorera y una progresiva disminución de las mismas para el marrajo. Dichas tendencias se realizaron con un esfuerzo pesquero relativamente estable hasta 2013, la disminución de la flota se observa sobre todo entre 2013 y 2016. El stock de tintorera no estaría sobreexplotado, pero la cantidad extraída de la biomasa del marrajo podría superar el RMS (Rendimiento Máximo Sostenible). Las autoridades a cargo de la pesca e instituciones (Ministerio de Pesca, Xunta de Galicia, ICCAT) deben tomar medidas para garantizar la explotación duradera de las especies.

\section{Agradecimientos}

El presente estudio se realizó gracias a la colaboración de los palangreros españoles y portugueses que han permitido el muestreo en 2012 y 2013. También los agradecimientos se dirigen a la autoridad portuaria de Vigo que han autorizado las fases de muestreo y a la Consejería del Mar de la Xunta de Galicia que han facilitado todos los datos estadísticos imprescindibles a la realización del trabajo presentado. El autor agradece a los 3 evaluadores anónimos que permitieron la publicación del estudio.

\section{LITERATURA CITADA}

Anón. 2009. Informe de la reunión de evaluación de stocks de tiburones de 2008. Collective Volume of Scientific Papers, ICCAT 64(5): 1343-1491.

Biton-Porsmoguer S. 2015. Biologie, écologie et conservation du requin peau bleue (Prionace glauca) et du requin mako (Isurus oxyrinchus) en Atlantique nord-est. Thèse de Doctorat, spécialité Océanographie, Aix-Marseille Université, Marseille, 269 pp.

Casey JG \& NE Kohler. 1992. Tagging studies on the shortfin mako shark Isurus oxyrinchus in the Western North Atlantic. Australian Journal of Marine and Freshwater Research 43: 45-60.

Clarke MR. 1986. A handbook for the identification of cephalopod beaks, 273 pp, Clarendon Press, Oxford.

Compagno L, M Dando \& S Fowler. 2005. Guía de campo de los tiburones del mundo, 357 pp. Ediciones Omega, Barcelona.

Figueiras FG, U Labarta \& MJ Fernández-Reiriz. 2002. Coastal upwelling, primary production and mussel growth in the Rías Baixas of Galicia. Hidrobiologia 484: 121-131.

Fromentin JM. 2006. Chapitre 2.1.5. Thon rouge de l'Atlantique (Thunnus thynnus). In: Manuel de l'ICCAT, pp. 99-119. <https://www.iccat.int/Documents/SCRS/ Manual/CH2/2_1_5_BFT-fra.pdf>

ICCAT. 2014-2015. 8.13 SHK-TIBURONES. Informe de Reuniones ICCAT 2014-2015 (II). International Commission for the Conservation of Atlantic Tuna. ICCAT publication on line. <https://www.iccat.int/Documents/ SCRS/ExecSum/SHK_SPA.pdf>

ICCAT. 2017. SMA Data Preparatory Meeting. Report of the 2017 Shortfin mako data prepatory meeting. International Commission for the Conservation of Atlantic Tuna. ICCAT publication on line. <https://www.iccat.int/Documents/ Meetings/Docs/2017_SMA_DATA_PREP_ENG.pdf >

Kirkley J, PCJ Morrison, S Cunningham \& J Catanzano. 2004. Embodied and disembodied technical change in fisheries: an analysis of the Sète trawl fishery, 1985-1999. Environmental and Resource Economics 29: 191-217.

Maia A, N Queiroz, J Correira \& H Cabra. 2006. Food habits of the shortfin mako, Isurus oxyrinchus, off the southern coast of Portugal. Environmental Biology of Fishes 77: 157-167.

Marchal P. 2008. A comparative analysis of métiers and catch profiles for some French demersal and pelagic fleets. ICES Journal of Marine Science 65: 674-686.

McCord ME \& SE Campana. 2003. A quantitative assessment of the diet of the blue shark (Prionace glauca) off Nova Scotia, Canada. Journal of Northwest Atlantic Fishery Science 32: 57-63. 
Mejuto J. 1985. Associated catches of sharks, Prionace glauca, Isurus oxyrinchus, and Lamna nasus with NW and $\mathrm{N}$ Spanish swordfish fishery, in 1984. ICES, Council Meeting 1985/H 42: 1-16.

Mejuto J \& AG Garcés. 1984. Shortfin mako, Isurus oxyrinchus, and porbeagle, Lamna nasus, associated with longline swordfish fishery in NW and N Spain. ICES CM Doc. G72: 1-10.

Mendonça A. 2009. Diet of the blue shark, Prionace glauca, in the Northeast Atlantic, 31 pp. Departamento de Biologia, Facultade de Ciências, Universidade do Porto, Porto.

Moreno JA. 2004. Guía de los tiburones de aguas ibéricas, Atlántico oriental y Mediterráneo, 315 pp. Edición Omega, Barcelona.

Muñoz-Chápuli R. 1984. Ethologie de la reproduction chez quelques requins de l'Atlantique nord-est. Cybium 8(4): 114.

Scherrer B. 2007. Biostatistique, 807 pp. Gaëtan Morin Éditeur, Chicoutimi.

Sobrinho-Gonçalves L \& E Isidro. 2001. Fish larvae and zooplankton biomass around Faial Island (Azores archipelago). A preliminary study of species occurrence and relative abundance. Arquipélago. Life and Marine Sciences 18A: 35-52.
Stevens JD. 1976. First results of shark tagging in the Northeast Atlantic, 1972-1975. Journal of the Marine Biological Association of the United Kingdom 56: 929-937.

Valeiras J \& E Abad. 2010a. Tintorera (Prionace glauca). Manual de ICCAT, pp. 259-269. Comisión Internacional para la Conservación del Atún Atlántico. <https:// www.iccat.int/Documents/SCRS/Manual/CH2/ 2_2_1_1_BSH_SPA.pdf>

Valeiras J \& E Abad. 2010b. Marrajo (Isurus oxyrinchus) Manual de ICCAT, pp. 271-281. Comisión Internacional para la Conservación del Atún Atlántico. <https:// www.iccat.int/Documents/SCRS/Manual/CH2/ 2_2_1_2_SMA_SPA.pdf>

Vandeperre F, A Aires-da-Silva, J Fontes, M Santos, $\mathbf{R}$ Serrão-Santos \& A Pedro. 2014. Movements of blue sharks (Prionace glauca) across their life history. PLOS ONE 9(8): 1-14.(e103538). <doi:10.1371/journal.pone.0103538>

Vasco Ferreira LMS. 2011. Guia de campo. Fauna e flora marinha de Portugal, 265 pp. Planeta Vivo, Leça da Palmeira.

Venables WN \& CM Dichmont. 2004. GLMs, GAMs and GLMMs: An overview of theory for applications in fisheries research. Fisheries Research 70: 319-337.

Recibido el 21 de noviembre de 2016 y aceptado el 31 de enero de 2018

Editor: Dr. Mauricio Landaeta D. 


\section{Anexo}
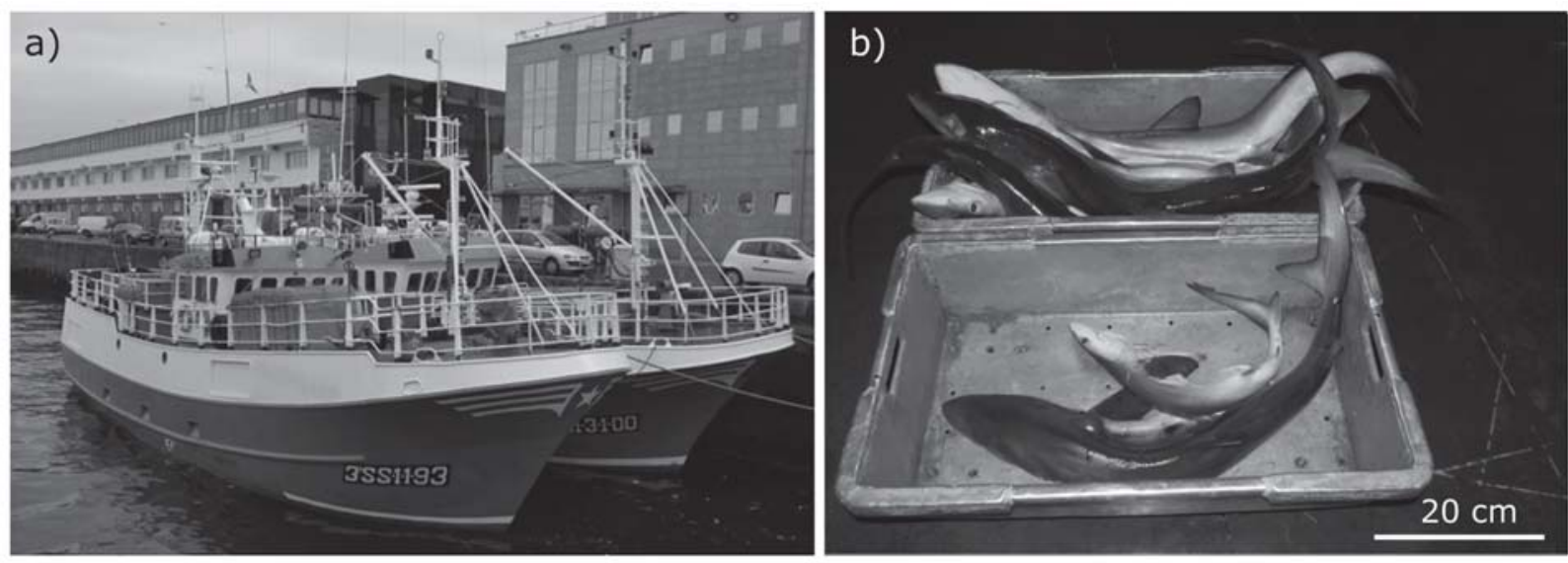

Figura S1. a) Palangreros españoles amarrados en el puerto de Vigo, marzo 2012, b) Juveniles de tintorera para venta en la lonja de Vigo (España). Octubre 2012. Fotografía: Sebastián Biton-Porsmoguer / a) Spanish longliners moored at the port of Vigo, March 2012, b) Juveniles of Blue sharks for sale in the fish market of Vigo (Spain). October 2012. Photography: Sebastián Biton-Porsmoguer 